\title{
Controlador robusto para el seguimiento de trayectorias para un exoesqueleto de extremidades inferiores
}

\section{Robust trajectory tracking controller for lower extremity exoskeleton}

\author{
BLANCO-ORTEGA, Andrés ${ }^{1} \dagger^{*}$, PEREZ-VIGUERAS, Demetrio ${ }^{1}$, ANTUNEZ-LEYVA, Edgar ${ }^{2}$ у \\ COLIN-OCAMPO, Jorge ${ }^{1}$
}

\author{
${ }^{I}$ Tecnológico Nacional de México/Centro Nacional de Investigación y Desarrollo Tecnológico \\ ${ }^{2}$ Instituto Mexicano de Tecnología del Agua
}

ID 1 ${ }^{\text {er }}$ Autor: Andrés, Blanco-Ortega / ORC ID: 0000-0002-0088-6863, CVU CONACYT ID: 123590

ID $1^{\text {er }}$ Coautor: Demetrio, Pérez-Vigueras / ORC ID: 0000-0002-7232-2039, CVU CONACYT ID: 599637

ID $2^{\text {do }}$ Coautor: Edgar, Antúnez-Leyva / ORC ID: 0000-0001-9630-1614

ID $3^{\text {er }}$ Coautor: Jorge, Colín Ocampo / ORC ID: 0000-0001-5579-0175, CVU CONACYT ID: 121050

\begin{abstract}
Resumen
Actualmente, la robótica ha demostrado que puede incrementar la eficiencia en la rehabilitación de los miembros del cuerpo humano, en este caso auxiliando a personas que han sufrido un accidente cerebrovascular (ACV), mediante el uso de dispositivos como los exoesqueletos, para proporcionar movimientos suaves y controlados. El accidente cerebrovascular es el resultado de una escasez de flujo sanguíneo al cerebro que conduce en apenas unos minutos a la muerte celular, ocasionando daños severos al cuerpo humano, inclusive puede causar la muerte del paciente. Personas que han sufrido un ACV presentan dificultades para levantarse de una silla. Un exoesqueleto es una estructura mecánica diseñada para ser usada sobre el cuerpo humano como una prenda de vestir, sirve de apoyo y es usado para asistir movimientos o acentuar la fuerza como apoyo en una persona. En este artículo se propone el uso de un controlador Proporcional Integral Generalizado (GPI) robusto para el seguimiento de trayectorias en un exoesqueleto de seis grados de libertad, para asistir a pacientes al levantarse y sentarse de una silla. Se presentan resultados obtenidos de simulaciones con el prototipo virtual del exoesqueleto, bajo el ambiente del software MSC Adams en cosimulación con Matlab
\end{abstract}

Exoesqueleto de extremidades inferiores, Control Robusto, Control GPI

\begin{abstract}
Currently, robotics has shown that it can increase the efficiency in the specific rehabilitation of some of the limbs of the human body, in this case assisting people who have suffered a stroke, by using devices such as exoskeletons, to provide continuous, smooth and controlled movements. Stroke is the result of a shortage of the brain that leads in just a few minutes to cell death, causing severe damage to the human body, even death of the patient. People who have suffered a stroke have difficulty rising from a chair. An exoskeleton is a mechanical structure designed to be used on the human body as a garment, it serves as a support and is used to assist movements or accentuate strength as a support in a person. Lower extremity exoskeleton can assist patients with hemiplegia to get up or sit on a chair, thus avoiding muscle atrophy and possible spasticity. This paper proposes the use of a robust Generalized Proportional Integral (GPI) controller for trajectory tracking for controlling a six degrees of freedom exoskeleton, to assist patients when getting up and sitting down from a chair. Simulation results obtained with the virtual prototype of the exoskeleton, under the environment of the MSC Adams software in co-simulation with Matlab are presented.
\end{abstract}

Lower extremity exoskeleton, Robust Control, GPI Control

Citación: BLANCO-ORTEGA, Andrés, PEREZ-VIGUERAS, Demetrio, ANTUNEZ-LEYVA, Edgar y COLIN-OCAMPO, Jorge. Controlador robusto para el seguimiento de trayectorias para un exoesqueleto de extremidades inferiores. Revista de Ingeniería Mecánica. 2019. 3-11: 1-8

\footnotetext{
* Correspondencia al Autor (Correo electrónico: andres.blanco@ cenidet.edu.mx)

$\uparrow$ Investigador contribuyendo como primer Autor.
} 


\section{Introducción}

El accidente cerebrovascular (ACV) es el resultado de una reducción de flujo sanguíneo al cerebro que conduce a la muerte celular, ocasionando daños severos al cuerpo humano, inclusive puede ocasionar hasta la muerte del paciente. Un ACV ocurre cuando un coágulo bloquea el flujo de sangre al cerebro o cuando se rompe un vaso sanguíneo en el cerebro. Las enfermedades cardiovasculares (ECV) y los $\mathrm{ACV}$ producen inmensos gastos económicos y de salud en todo el mundo. En 2016 el ACV fue la segunda causa de mortalidad en los Estados Unidos, y son una causa importante de discapacidad que reducen la movilidad en más de la mitad de los sobrevivientes de 65 años y mayores (Benjamin, 2019) (Rehmat, 2018) (Zhi, 2018).

Los sobrevivientes que han sufrido un accidente cerebrovascular (ACV) generalmente sufren de hemiplejia (parálisis de un lado del cuerpo) o pérdida total de movimiento, llegando a ser hasta un $80 \%$ de los sobrevivientes de un ACV (Ochoa, 2015). El ACV afecta en gran medida la capacidad del paciente para realizar trabajos y actividades de la vida cotidiana ( $\mathrm{Zhi}$, 2018). Las personas con debilidad en las extremidades inferiores tienen dificultades para moverse o realizar actividades de la vida diaria sin el apoyo de un asistente. Ayudar a estas personas a ponerse de pie, sentarse y caminar es una tarea ardua y pone al asistente bajo presión, lo que puede provocar caídas y causar lesiones tanto al paciente como al asistente.

El ACV y otras enfermedades y lesiones (bursitis, osteoporosis, traumatismos óseos y musculares, entre otras) que afectan las extremidades inferiores provoca que el paciente se someta a un proceso de rehabilitación para evitar la rigidez, espasticidad, disminución del tono muscular, pérdida de extensibilidad $\mathrm{y}$, en caso contrario, recuperar el rango de movimiento.

Mantener en movimiento el cuerpo o realizar alguna práctica deportiva presenta varios beneficios, entre ellos: la oxigenación del organismo, liberar energía, mejorar la circulación sanguínea, prevenir la obesidad, disminuir la tensión arterial, preservar el tono muscular y ayuda a combatir la depresión, entre otros beneficios.
Por eso es importante que el paciente, mediante un proceso de rehabilitación, recupere sus funciones normales que tenía antes de haber sufrido la lesión. Los especialistas en fisioterapia realizan un esfuerzo grande en el proceso de rehabilitación, ya que tienen que cargar la extremidad del paciente para realizar los movimientos. La calidad de los movimientos va decayendo conforme se atienden los pacientes. Además, cada sesión de rehabilitación es de media a $1 \mathrm{~h}$.

Atendiendo a esta problemática, se han propuesto diversos dispositivos mecatrónicos para ayudar al paciente a realizar los movimientos básicos y vitales: levantarse, sentarse y caminar. Estos dispositivos, conocidos como exoesqueletos, permiten a sus usuarios superar las dificultades asociadas con los movimientos. Para realizar dichas actividades de forma independiente, estos sistemas deben ser robots portátiles que brinden potencia, soporte y equilibrio debido a la limitación o falta de control sobre sus miembros inferiores.

Un exoesqueleto es una estructura mecánica diseñada para ser usada sobre el cuerpo humano como una prenda de vestir, sirve de apoyo y es usado para asistir movimientos o aumentar la fuerza en un ser humano (Lee, 2014) (Marcheschi, 2011).

Los exoesqueletos robóticos constituyen formas semi-naturales de ejercitar el cuerpo y las extremidades afectadas. Proporcionan a sus usuarios muchos beneficios sobre la terapia de rehabilitación tradicional. Como se mencionan a continuación (Ghaddar, 2019):

- Los exoesqueletos brindan una marcha segura en entornos del mundo real que producen beneficios para la salud.

- El ejercicio de las extremidades afectadas conserva la masa muscular.

Restauración de las funciones sensoriales y musculares.

- Son dispositivos factibles para mejorar las habilidades de caminar y mantener el equilibrio.

- Las demandas cardiorrespiratorias y metabólicas al usar un exoesqueleto son equivalentes a las actividades realizadas a una intensidad moderada.

- $\quad$ Aceleran el período de rehabilitación del paciente para obtener control y equilibrio del cuerpo.

BLANCO-ORTEGA, Andrés, PEREZ-VIGUERAS, Demetrio, ANTUNEZ-LEYVA, Edgar y COLIN-OCAMPO, Jorge. Controlador robusto para el seguimiento de trayectorias para un exoesqueleto de extremidades inferiores. Revista de Ingeniería Mecánica. 2019 
- $\quad$ El sentido de independencia, que eleva la calidad de vida del paciente.

La reducción de las complicaciones secundarias de salud que pueden producirse si el paciente sufre de movilidad, como la obesidad, la atrofia muscular y la osteoporosis.

A continuación, se mencionan algunos exoesqueletos enfocados a la asistencia de la acción de sentarse-levantarse. En (Qureshi, 2018) presentan un exoesqueleto robótico de miembros inferiores que puede ayudar a las personas paralizadas. El exoesqueleto proporciona 3 gdl por extremidad, de los cuales dos articulaciones están activas y una pasiva. Los autores implementan un control de par calculado - PID para los movimientos de estar sentado para estar de pie y de pie para estar sentado.

Las ganancias del controlador PID son diferentes en la acción de sentarse-pararse a la de pararse-sentarse. Aunque presentan un movimiento suave en el control del exoesqueleto, éste no está basado en la biomecánica del ser humano. No reportan el torque requerido. Otro exoesqueleto, para la transición de sentarse-levantarse, denominado H1 es presentado en (Rajasekaran, 2017). Los autores proponen un control basado en eventos (control acoplado de amortiguamiento-rigidez), con un perfil de velocidad trapezoidal para la transición. Reportan resultados de simulación sin considerar la biomecánica del ser humano en la transición de sentarse-levantarse, dónde se requiere cerca de $40 \mathrm{Nm}$ en las uniones de la cadera y rodilla. También, reportan resultados del exoesqueleto con pacientes sanos, con un tiempo en la transición de 1 a $3 \mathrm{~s}$ y torque máximo de $20 \mathrm{Nm}$ en la rodilla.

\section{Desarrollo}

\section{Descripción del sistema}

En la Fig. 1 se muestra el diagrama esquemático para representar la extremidad inferior mediante tres eslabones conectados en serie y un eslabón para representar el pie. Dónde los ángulos para cada eslabón están denotados por $\theta_{1}$ (pierna), $\theta_{2}$ (muslo) y $\theta_{3}$ (cadera). Las entradas de control están denotadas por $\tau_{1}, \tau_{2}$ y $\tau_{3}$. Para cada segmento de la extremidad $(i=1,2,3)$ se considera su momento de inercia $\left(I_{i}\right)$, masa $\left(m_{i}\right)$ y longitud $\left(l_{i}\right)$.
El modelo dinámico fue reportado en (Pérez, 2018), así como un análisis de la biomecánica en la transición de sentarse-pararse.

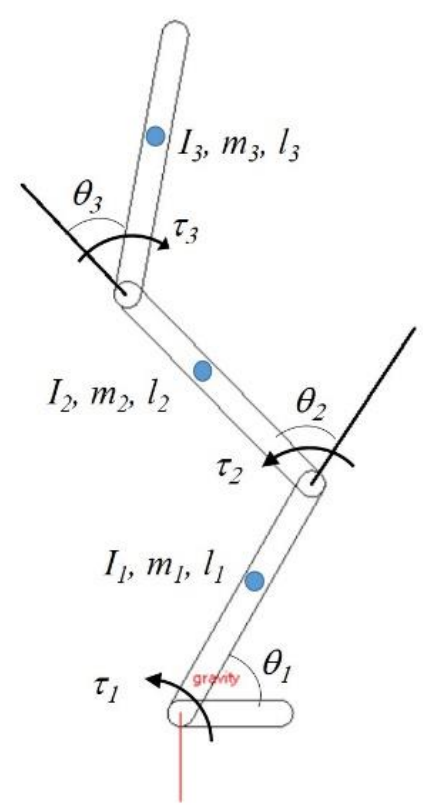

Figura 1 Diagrama esquemático del exoesqueleto Fuente: Elaboración Propia (2019)

Para las simulaciones que se presentan en este artículo se considera el diseño de la estructura del exoesqueleto mostrado en la Fig. 2. Como se puede apreciar, se consideran las fuerzas que ejerce cada segmento de las extremidades inferiores del usuario, considerando el peso de un usuario de $80 \mathrm{~kg}$. El punto de aplicación de cada fuerza es considerando el centro de masa de cada segmento, y siempre actúa en la dirección vertical.

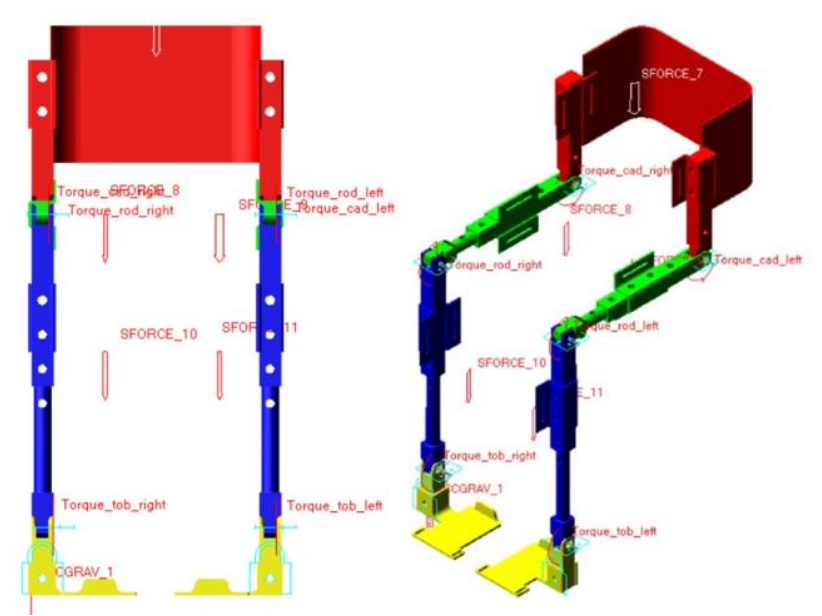

Figura 2 Exoesqueleto con fuerzas denotando el peso de cada segmento de las extremidades del usuario Fuente: Elaboración Propia (2019)

Considerando la Fig. 1 y 2 , los parámetros del exoesqueleto se muestran en la tabla 1. Dónde $l_{i g}(i=1,2,3)$ es la longitud de la articulación $i$ al centro de masa del eslabón $i$. 


\begin{tabular}{|l|r|r|r|}
\hline \multicolumn{1}{|c|}{$\mathbf{i}$} & \multicolumn{1}{c}{$\mathbf{1}$} & \multicolumn{1}{c|}{$\mathbf{2}$} & \multicolumn{1}{c|}{$\mathbf{3}$} \\
\hline$I_{i}\left(\mathrm{kgm}^{2}\right)$ & $1.78 \times 10^{-2}$ & $7.67 \times 10^{-3}$ & $2.69 \times 10^{-2}$ \\
\hline$m_{i}(\mathrm{~kg})$ & 1.0 & 0.91 & 1.4 \\
\hline$l_{i}(\mathrm{~m})$ & 0.45 & 0.49 & 0.25 \\
\hline$l_{i g}(\mathrm{~m})$ & 0.25 & 0.32 & 0.15 \\
\hline
\end{tabular}

Tabla 1 Parámetros de simulación del prototipo virtual del exoesqueleto

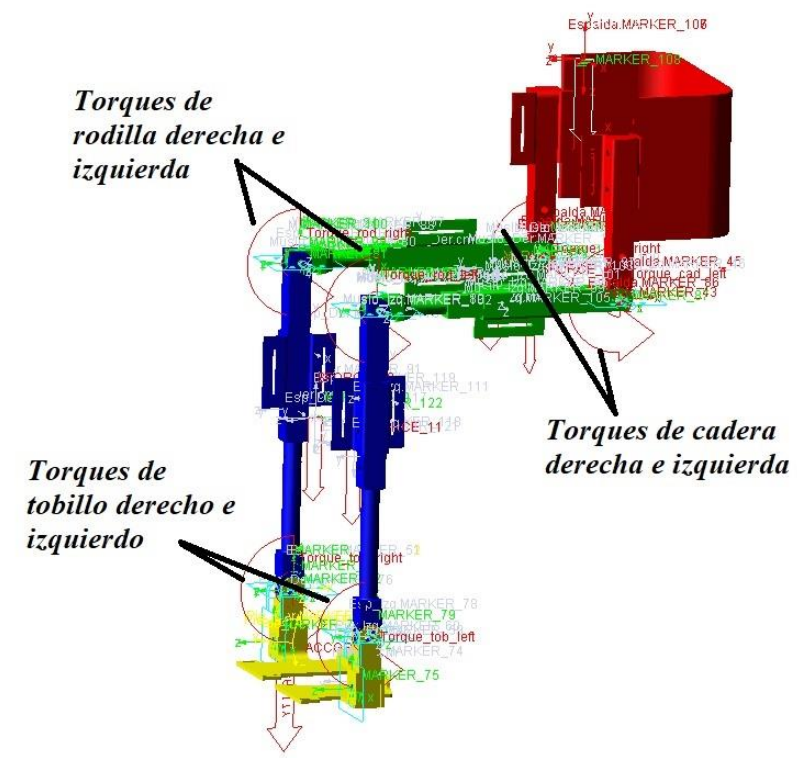

Figura 3 Entradas de control en cada eslabón del prototipo virtual

Fuente: Elaboración Propia (2019)

Para las simulaciones con el prototipo virtual, se considera un paciente con peso promedio de $70 \mathrm{~kg}$. El peso de cada segmento se determinó con base en los porcentajes reportados por Dempster y Clauser (Diego-Mas, 2015).

\begin{tabular}{|l|r|r|}
\multicolumn{4}{|c|}{ Porcentajes de masa por segmento para un paciente de } \\
\multicolumn{1}{|c|}{ Segmento } & \multicolumn{1}{c|}{ Masa (\%) } & \multicolumn{1}{c|}{ Peso neto } \\
\hline Cabeza y cuello & 6.94 & 4.858 \\
\hline Tronco & 43.46 & 30.422 \\
\hline Brazo & 2.71 & 1.897 \\
\hline Antebrazo & 1.62 & 1.134 \\
\hline Mano & 0.61 & 0.427 \\
\hline Muslo & 14.17 & 7.0 \\
\hline Pantorrilla & 4.33 & 3.26 \\
\hline Pie & 1.37 & 0.959 \\
\hline
\end{tabular}

Tabla 2 Parámetros de simulación del prototipo virtual del exoesqueleto

\section{Diseño de controlador GPI robusto}

Para el control del exoesqueleto, se implementa un controla activo de uniones, debido a que diseñar una estrategia de control a partir del modelo matemático resulta complejo. Por lo que se controla de manera independiente cada segmento del exoesqueleto.
Para lograr el control y el seguimiento de una trayectoria suave se propone la implementación de un control robusto proporcional integral generalizado (GPI), de manera que compense las fuerzas acopladas de todo el sistema en la transición de sentarsepararse. Para el diseño de la ley de control robusto PI generalizado, se plantea el modelo matemático (1) que representa un sistema de un grado de libertad (gdl). En este caso para el primer eslabón (pierna derecha o izquierda):

$J_{e 1} \ddot{\theta}_{1}=\tau_{1}+P_{1}(t)$

Donde $J_{e l}$ representa el momento de inercia que debe de mover el torque de control $\tau_{1}, P_{1}$ representa los términos acoplados $\mathrm{y}$ perturbaciones presentes en dicha articulación. La perturbación es desconocida pero acotada y en este caso se considera que puede ser aproximada mediante un polinomio de tercer grado, dado por:

$P_{1}(t)=a t^{3}+b t^{2}+c t+d$

Se propone el siguiente controlador para el seguimiento de trayectorias y considerando la perturbación dependiente del tiempo tipo cúbica:

$\tau_{1}=\ddot{\theta}_{1 d}-k_{5}\left(\hat{\theta}_{1}-\dot{\theta}_{1 d}\right)-k_{4}\left(\theta-\theta_{1 d}\right)-k_{3} \int_{0}^{t}\left(\theta_{1}-\right.$

$\left.\theta_{1 d}\right) d \sigma-k_{2} \int_{0}^{t} \int_{0}^{\sigma}\left(\theta_{1}-\theta_{1 d}\right) d \lambda d \sigma-k_{1} \int_{0}^{t} \int_{0}^{\sigma} \int_{0}^{\lambda}\left(\theta_{1}-\right.$

$\left.\theta_{1 d}\right) d \xi d \lambda d \sigma-k_{0} \int_{0}^{t} \int_{0}^{\sigma} \int_{0}^{\lambda} \int_{0}^{\xi}\left(\theta_{1}-\theta_{1 d}\right) d \eta d \xi d \lambda d \sigma$

Donde (7) es el reconstructor integral de la velocidad del actuador.

$\hat{\hat{\theta}}_{1}=\int_{0}^{t} \tau_{1}(\psi) d \psi$

Sustituyendo (6) en (4), considerando el reconstructor integral y la perturbación tipo rampa, se tiene

$$
\begin{aligned}
& J_{e 1} \ddot{\theta}_{1}=\ddot{\theta}_{1 d}-k_{5}\left(\int_{0}^{t} \tau_{1}(\psi) d \psi-\dot{\theta}_{1 d}\right)-k_{4}\left(\theta_{1}-\theta_{1 d}\right)- \\
& k_{3} \int_{0}^{t}\left(\theta_{1}-\theta_{1 d}\right) d \sigma-k_{2} \int_{0}^{t} \int_{0}^{\sigma}\left(\theta_{1}-\theta_{1 d}\right) d \lambda d \sigma- \\
& k_{1} \int_{0}^{t} \int_{0}^{\sigma} \int_{0}^{\lambda}\left(\theta_{1}-\theta_{1 d}\right) d \xi d \lambda d \sigma-k_{0} \int_{0}^{t} \int_{0}^{\sigma} \int_{0}^{\lambda} \int_{0}^{\xi}\left(\theta_{1}-\right. \\
& \left.\theta_{1 d}\right) d \eta d \xi d \lambda d \sigma+a t^{3}+b t^{2}+c t+d
\end{aligned}
$$

Definiendo el error como $e=\theta_{1}-\theta_{l d}, \mathrm{y}$ derivando dos veces se obtiene la ecuación dinámica del error dada por:

$e^{V I}+k_{5} e^{V}+k_{4} e^{I V}+k_{3} \dddot{e}+k_{2} \ddot{e}+k_{1} \dot{e}+$
$k_{0} e=0$ 
Aplicando transformada de Laplace a (6), se obtiene la ecuación característica del sistema en lazo cerrado:

$$
\begin{aligned}
& s^{6}+k_{5} s^{5}+k_{4} s^{4}+k_{3} s^{3}+k_{2} s^{2}+k_{1} s+ \\
& k_{0}=0
\end{aligned}
$$

Las ganancias del controlador $\left(k_{i}\right.$, $i=0,1, \ldots, 5)$ se determinan al igualar a un polinomio Hurwitz para que la dinámica del error sea asintóticamente estable, dado por:

$\left(s^{2}+2 \zeta \omega s+\omega^{2}\right)^{3}=0$

Para este caso se seleccionaron:

$\zeta=1.5$ y $\omega=8$

\section{Trayectoria de referencia}

Para evitar un movimiento brusco en las etapas de la transición sentado - levantarse, se utilizó un polinomio de Bézier, dado por (8). Para iniciar en un tiempo $t_{i}$ desde un ángulo inicial $\theta_{i}$, para llevar el eslabón de manera suave a un valor final de $\theta_{f}$ en un tiempo final $t_{f}$.

$\theta_{d}(t)=\theta_{i}+\left(\theta_{f}-\theta_{i}\right) \sigma\left(t, t_{i}, t_{f}\right) \mu_{p}^{5}$

$\sigma\left(t, t_{i}, t_{f}\right)=\gamma_{0}-\gamma_{1} \mu_{p}+\gamma_{2} \mu_{p}^{2}-\cdots+\gamma_{5} \mu_{p}^{5}$

$\mu_{p}=\frac{t-t_{i}}{t_{f}-t_{i}}$

Los parámetros del polinomio son $\gamma_{1}=252, \gamma_{2}=1050, \gamma_{3}=1800, \gamma_{4}=1575, \gamma_{5}=700 \mathrm{y}$ $\gamma_{6}=126$.

Los tiempos y valores inicial y final para cada eslabón del exoesqueleto durante la transición de sentado-pararse, se muestran en la Tabla 3.

\begin{tabular}{|l|r|r|r|r|}
\cline { 2 - 5 } \multicolumn{1}{c|}{} & \multicolumn{1}{c|}{$t_{i}(\mathrm{~s})$} & $\theta_{i}\left({ }^{\circ}\right)$ & $t_{f}(\mathrm{~s})$ & $\theta_{f}\left({ }^{\circ}\right)$ \\
\hline Pierna & 0 & 90 & 2 & 90 \\
\hline \multirow{2}{*}{ Muslo } & 0 & 90 & 0.3 & 85 \\
\cline { 2 - 5 } & 0.3 & 85 & 2 & 0 \\
\hline \multirow{2}{*}{ Cadera } & 0 & 90 & 0.4 & 107 \\
\cline { 2 - 5 } & 0.3 & 107 & 2 & 0 \\
\hline
\end{tabular}

Tabla 3 Parámetros de simulación del prototipo virtual del exoesqueleto

\section{Resultados}

Los resultados que se presentan a continuación se realizaron en MSC Adams ${ }^{\circledR}$ en co-simulación con Matlab-simulink®. Como se mencionó anteriormente, se utilizará el polinomio de Bezier (ecuación (8)) para llevar el pacienteexoesqueleto desde una posición inicial de sentado a una final de parado.

Para las pruebas de validar el desempeño del controlador GPI robusto, en cada articulación del exoesqueleto, para realizar la transición de sentado-parado se considera siempre las fuerzas que ejerce el usuario en el exoesqueleto debido al peso de cada segmento, considerando los valores de masa indicados en la Tabla 2.

Se realiza una primera simulación (caso 1) en la que se considera una condición, dónde el ángulo de la articulación de los tobillos $\left(\theta_{1}\right)$ siempre se mantiene en $90^{\circ}$. Además, se considera también que el peso de las manos, brazo, antebrazo, tronco, cabeza y cuello actúan en centro de masa del tronco.

En la segunda simulación (caso 2) se considera un movimiento natural del humano, es decir, se consideran los valores obtenidos del análisis biomecánico reportado en (Demetrio, 2018). En este caso, se puede observar que los centros de masa de los eslabones tratan de actuar directamente en el punto de apoyo para evitar momentos que requieran un mayor esfuerzo en las entradas de control (torques en articulaciones).

\section{Caso 1}

Para el primer caso, se muestra en la Figura 4 los ángulos de cada uno de los eslabones. Considerando que el movimiento es simétrico, se tiene la misma respuesta para ambas piernas (derecha e izquierda), por lo que solo se presenta la respuesta para cada ángulo de la pierna $\left(q_{1}=\theta_{1}\right)$, muslo $\left(q_{2}=\theta_{2}\right)$ y cadera $\left(q_{3}=\theta_{3}\right)$. En la respuesta de las posiciones angulares se presenta tanto la trayectoria deseada como la obtenida con el controlador GPI robusto, como se aprecia, se tiene un buen desempeño en el seguimiento de las trayectorias. El tiempo en que se realiza la transición de sentado-parado es de $2 \mathrm{~s}$, como se indica en la Tabla 3 y se muestra en la Figura 4. 
Los tiempos reportados en la literatura (Mughal, 2010) para la transición de sentadoparado $1.907 \pm 0.0507 \mathrm{~s}$ y de $1.7 \mathrm{a} 2.3 \mathrm{~s}$. Se puede observar que el control $\tau_{1}$ mantiene el eslabón de la pierna en $90^{\circ}$. También, se puede apreciar que en el torque aplicado en la rodilla es el más grande, aproximadamente $150 \mathrm{Nm}$, similar al reportado por (Mughal, 2010) de $180 \mathrm{Nm}$. Es importante mencionar que el peso del paciente se considera como si no aportará fuerzas adicionales para ayudar en la transición de sentado-parado, es decir, como si se dejará caer para que el exoesqueleto lo levante de la silla hasta ponerlo de pie.
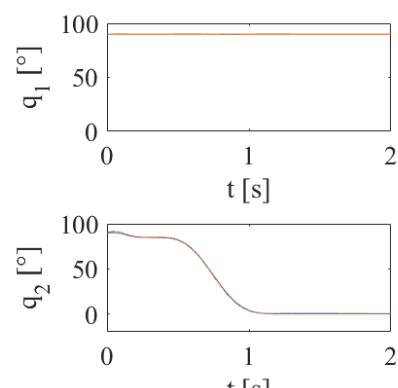

$\mathrm{t}[\mathrm{s}]$

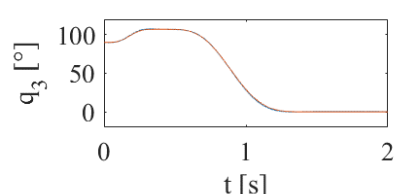

$\mathrm{t}[\mathrm{s}]$

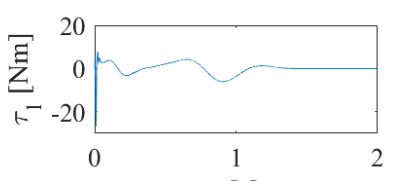

$\mathrm{t}[\mathrm{s}]$

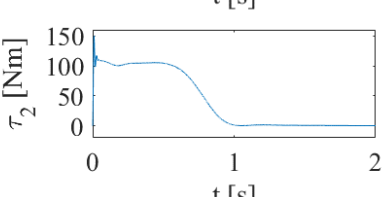

$\mathrm{t}[\mathrm{s}]$

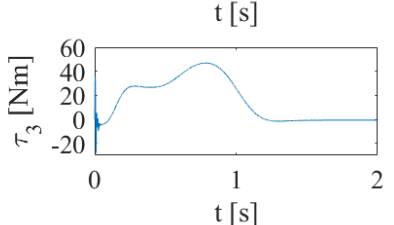

Figura 4 Respuesta de las posiciones angulares y toques requeridos en cada uno de los eslabones del exoesqueleto Fuente: Elaboración Propia (2019)

En la Fig. 5 se muestra la secuencia de la transición del exoesqueleto de sentado a parado. La secuencia de cada imagen es de $0.3 \mathrm{~s}$, aproximadamente. Se puede notar que el eslabón de la pierna se mantiene siempre a $90^{\circ}$, un caso inusual en el movimiento natural del humano, sin embargo, con el exoesqueleto es posible realizarlo; mostrando que bajo esta condición puede levantar al paciente con los torques resultantes mostrados en la Fig. 4.
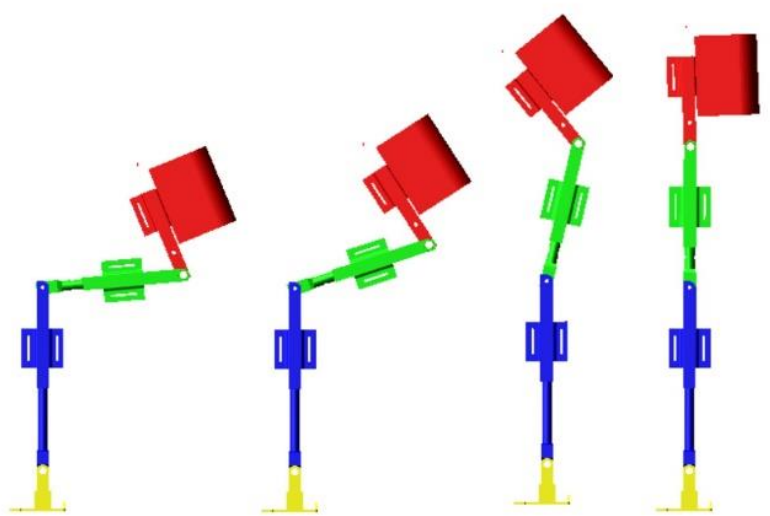

Figura 5 Secuencia de la posición angular en la transición de sentado-parado para posicionar verticalmente el exoesqueleto

Fuente: Elaboración Propia (2019)

\section{Caso 2}

En la segunda simulación, se consideran los tiempos y posiciones angulares obtenidos del análisis biomecánico (Pérez, 2018), ver Tabla 3, considerando una posición inicial de sentado, posteriormente, pasar a una posición para iniciar el levantamiento hasta llegar a una posición de parado.

Para lograr este movimiento más natural, se propone utilizar 3 polinomios para la pierna, muslo y cadera, considerado los tiempos y ángulos indicados en la Tabla 3. Para esta transición se observa que se realizará en 2.3s.

\begin{tabular}{|l|r|r|r|r|}
\hline \multirow{4}{*}{ Pierna } & \multicolumn{1}{|c}{$t_{i}(\mathrm{~s})$} & $\theta_{i}\left({ }^{\circ}\right)$ & \multicolumn{1}{c|}{$t_{f}(\mathrm{~s})$} & $\theta_{f}\left({ }^{\circ}\right)$ \\
\cline { 2 - 5 } & 0 & 90 & 0.4 & 82 \\
\cline { 2 - 5 } & 0.4 & 82 & 1.3 & 72 \\
\hline \multirow{4}{*}{ Muslo } & 1.3 & 72 & 2.3 & 84 \\
\cline { 2 - 5 } & 0 & 90 & 0.4 & 90 \\
\hline \multirow{4}{*}{ Cadera } & 0.4 & 90 & 1.3 & 75 \\
\cline { 2 - 5 } & 1.3 & 75 & 2.3 & 4 \\
\cline { 2 - 5 } & 0.4 & 90 & 0.4 & 107 \\
\cline { 2 - 5 } & 1.3 & 107 & 2.3 & 4 \\
\hline
\end{tabular}

Tabla 3 Parámetros de simulación del prototipo virtual del exoesqueleto

En la Figura 6, se muestra la respuesta utilizando el controlador GPI robusto, se aprecia un buen desempeño en el seguimiento de las trayectorias deseadas. En este caso, se observa que se requiere un mayor par en el tobillo comparado al caso anterior, Figura 4.
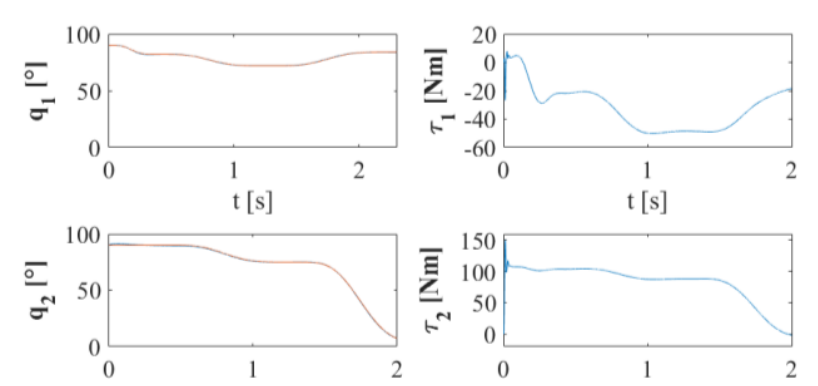

$\stackrel{1}{t[s]}$

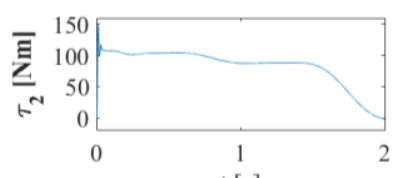

$\mathrm{t}[\mathrm{s}]$
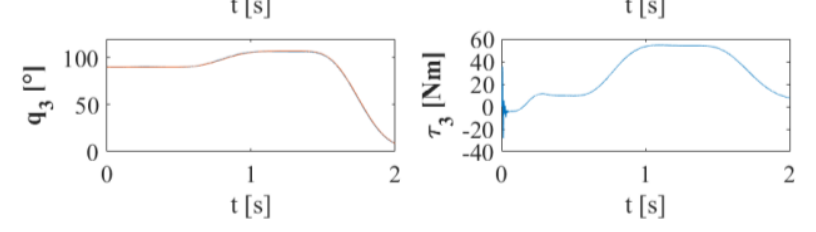

Figura 6 Respuesta de las posiciones angulares y toques requeridos en cada uno de los eslabones del exoesqueleto basado en tiempos de un movimiento natural Fuente: Elaboración Propia (2019) 
En la Fig. 7 se muestra la secuencia de movimientos en la transición de sentado-parado. Se puede observar que en la primera etapa se pone en posición inicial $\left(\theta_{l}=82^{\circ}\right)$ para iniciar el levantamiento. En esta etapa la pierna no se encuentra a $90^{\circ}$. Se inclina la cadera-tronco y se inicia el movimiento en la articulación de las rodillas. Se observa en las siguientes etapas que, si se considera una resultante de todos los pesos, está actúa directamente en la dirección de la articulación de los tobillos, evitando así generar momentos que pudieran requerir una compensación.

La ventaja de este controlador GPI robusto (3) es que solo requiere de mediciones de la salida, es decir, solo mediciones del ángulo a controlar. Por lo que, para su implementación se requiere de 6 sensores de posición angular.

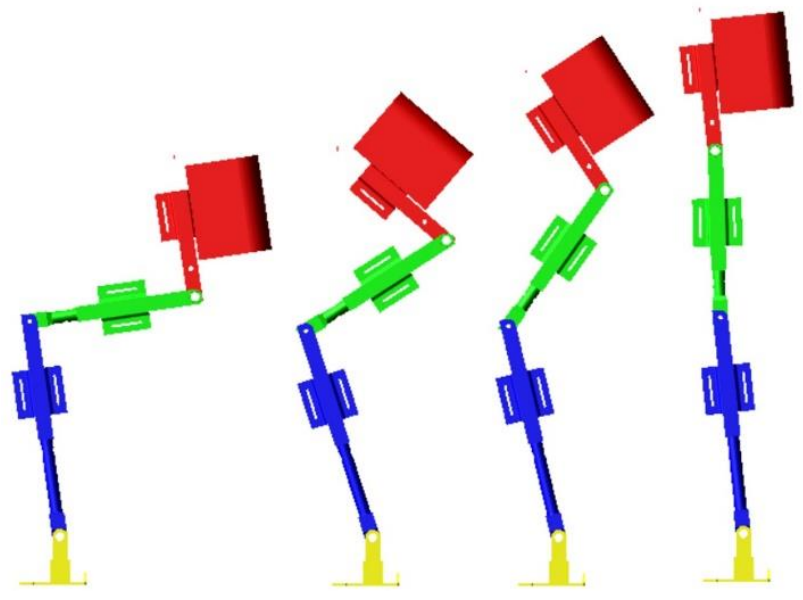

Figura 7 Secuencia de la posición angular en la transición de sentado-parado siguiendo trayectorias naturales Fuente: Elaboración Propia (2019)

Como se observa en la Figura 7, los eslabones no quedan totalmente verticales como se muestra en la Figura 5, esto es debido a la posición natural del humano. Esta secuencia se puede apreciar de manera similar a la reportada en (Rajasekaran, 2017), donde un usuario utiliza un exoesqueleto de extremidades inferiores. También, es importante mencionar que los valores obtenidos en estas simulaciones, son similares a los reportados por otros autores al analizar los torques en las articulaciones de una persona, cuando realiza la transición de sentadoparado, como se menciona en (Lara, 2015).

Se construyó el prototipo físico del exoesqueleto de extremidades inferiores, ver Figura 8, para validar los resultados obtenidos, faltando instrumentar para realizar las pruebas con el controlador GPI robusto.

\section{Agradecimiento}

Este trabajo ha sido realizado al amparo del Programa de Maestría en Ciencias de Ingeniería Mecánica, del Tecnológico Nacional de México/ CENIDET.

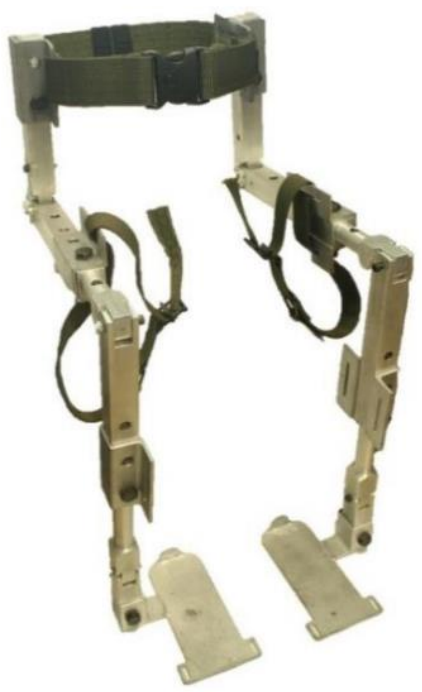

Figura 8 Prototipo físico de exoesqueleto de extremidades inferiores

Fuente: Elaboración Propia (2019)

\section{Conclusiones}

En este artículo se propone un controlador GPI robusto para el control de un exoesqueleto de extremidades inferiores en la transición de sentado-parado. El controlador tiene como objetivo el seguimiento de trayectorias, las cuales están basados en el análisis biomecánico (ángulos y tiempos) de la transición de levantarse y sentarse del ser humano. Los resultados de simulación muestran un buen desempeño de este controlador considerando las fuerzas que puede generar un usuario dejando caer su peso en el exoesqueleto. Como trabajo futuro se contempla su validación experimental.

\section{Referencias}

Benjamin E. J. et al., (2019). Heart Disease and Stroke Statistics 2019 Update. A Report from the American Heart Association. Circulation;139: e56-e528.

Diego-Mas, J. A. (2015). Análisis biomecánico estático coplanar. Ergonautas, Universidad Politécnica de Valencia. Disponible online: https://www.ergonautas.upv.es/metodos/biomec anica/biomecanica-ayuda.php 
Ghaddar R. and Mohammad A.S. (2019). A Review of Lower Limb Exoskeleton Assistive Devices for Sit-To-Stand and Gait Motion. International Journal of Current Engineering and Technology, Vol.9, No.1.

Lara-Barrios, C. M. Blanco-Ortega, A. Abúndez-Pliego, A. and Colín-Ocampo, J. (2015). Sit-to-Stand Simulation for Torque Estimation on Lower Limb Joints, 2015 International Conference on Mechatronics, Electronics and Automotive Engineering ICMEAE 2015, Prague, 116-121.

Lee H., Lee B., Kim W., Han J., Shin K., Han C. (2014). Human-robot cooperation control based on a dynamic model of an upper limb exoskeleton for human power amplification. Mechatronics, 24: 168-76.

Mughal, A. M. and Iqbal, K. (2010). 3D bipedal model for biomechanical sit-to-stand movement with coupled torque optimization and experimental analysis, 2010 IEEE International Conference on Systems, Man and Cybernetics, Istanbul, 568-573.

Ochoa Luna C, Habibur Rahman M, Saad M, Archambault P. S, \& Bruce Ferrer S (2015). Admittance-based upper limb robotic active and active-assistive movements. International Journal of Advanced Robotic Systems, 12(9), 117.

Rehmat, N., Zuo, J., Meng, W., Liu, Q., Xie, S.Q., Liang, H. (2018). Upper limb rehabilitation using robotic exoskeleton systems: a systematic review. Int J Intell Robot Appl. 2 (3), 283-295.

Marcheschi, S., Salsedo, F., Fontana, M. and Bergamasco, M. (2011). Body Extender: Whole body exoskeleton for human power augmentation," 2011 IEEE International Conference on Robotics and Automation, Shanghai, 2011, 611-616.

Pérez-Vigueras, D., Blanco-Ortega, A., Guzmán-Valdivia, C. H., Alcocer-Rosado, W. M. (2018). Análisis biomecánico de la acción sentarse-levantarse para el control de exoesqueletos. Revista de Ingeniería Biomédica y Biotecnología. 2-4: 34-44.
Qureshi, M. H. Masood, Z. Rehman, L. Owais, M. and Khan, M. U. (2018). Biomechanical Design and Control of Lower Limb Exoskeleton for Sit-to-Stand and Stand-to-Sit Movements, 14th IEEE/ASME International Conference on Mechatronic and Embedded Systems and Applications (MESA), Oulu, 1-6.

Rajasekaran, V., Vinagre, M. and Aranda, J. (2017). Event-based control for sit-to-stand transition using a wearable exoskeleton, 2017 International Conference on Rehabilitation Robotics (ICORR), London, 400-405.

Zhi, Y. X., Lukasik, M., Li, E. Dolatabadi, M. H. Wang, R. H. and Taati, B. (2018). Automatic Detection of Compensation During Robotic Stroke Rehabilitation Therapy, in IEEE Journal of Translational Engineering in Health and Medicine, vol. 6, 1-7. 